\section{Prevalência de pré-hipertensão e de hipertensão arterial e avaliação de fatores associados em crianças e adolescentes de escolas públicas de Salvador, Bahia, Brasil}

\author{
Prevalence of pre-hypertension and arterial \\ hypertension and evaluation of associated \\ factors in children and adolescents in public \\ schools in Salvador, Bahia State, Brazil
}

Sônia Lopes Pinto 1 Rita de Cássia Ribeiro Silva 1 Silvia Eloíza Priore 2 Ana Marlúcia Oliveira Assis 1 Elizabete de Jesus Pinto 1

\footnotetext{
1 Escola de Nutrição, Universidade Federal da Bahia, Salvador, Brasil. 2 Departamento de Nutrição e Saúde, Universidade Federal de Viçosa, Viçosa, Brasil.

Correspondência R. C. R. Silva Escola de Nutrição, Universidade Federal da Bahia.

Rua Araújo Pinho 32, Salvador, BA 40150-260, Brasil. rcrsilva@ufba.br
}

\section{Abstract}

This study aimed to assess pre-hypertension and hypertension-related factors in 1,125 sevento-14-year-old subjects enrolled in the public school system in Salvador, Bahia State, Brazil. Exposure variables, namely body mass index, waist circumference, gender, age, physical activity, environmental and housing status, family income, diet, schooling, and maternal age were analyzed by polytomous logistic regression, and the outcome variable was categorized as normal, pre-hypertensive, and hypertensive. Prevalence of high blood pressure was $14.1 \%$, including the prevalence of both hypertension (4.8\%) and prehypertension (9.3\%). An association was shown between pre-hypertension and overweight (OR: 3.13; 95\%CI: 1.75-5.57). Hypertension was associated with overweight (OR: 3.02; 95\% CI: 1.456.28), female gender (OR: 2.49; 95\%CI: 1.24-4.98), and high-risk eating patterns (OR: 1.93; 95\%CI: 1.04-3.56). In short, prevalence of pre-hypertension and hypertension in children and adolescents was higher among girls and individuals with overweight and inadequate diet.

Prehypertension; Hypertension; Risk Factors; Child; Adolescent

\section{Introdução}

Segundo as V Diretrizes Brasileiras de Hipertensão Arterial 1, a hipertensão arterial sistêmica (HAS) é definida como uma síndrome multifatorial caracterizada pela presença de níveis tensionais elevados, associados a alterações metabólicas, hormonais e fenômenos tróficos, os quais consistem na hipertrofia cardíaca e vascular. A HAS atinge cerca de $30 \%$ da população adulta no mundo, nos países desenvolvidos e nos em desenvolvimento 2 . No continente americano, a síndrome afeta cerca de 140 milhões de pessoas, metade das quais desconhece ser portadora da doença por não apresentar sintomas e não sentir a necessidade de procurar o serviço de saúde. Daquelas que se descobrem hipertensas, $30 \%$ não realizam o tratamento adequado por falta de motivação ou de acesso aos serviços de saúde 3 .

De acordo com a Sociedade Brasileira de Hipertensão, a prevalência nacional de hipertensão arterial sistêmica na população adulta varia de $22,3 \%$ a $43,9 \%$. Embora a maior parte dos diagnósticos de hipertensão arterial sistêmica seja firmada em pacientes com idade avançada, existem evidências de que a doença tem seu início na infância ou na adolescência 4,5. A pressão arterial elevada na infância é fator preditor de hipertensão arterial na vida adulta; portanto, o aumento da doença na infância pode significar o aumento de HAS nos adultos 6 . 
No Brasil, não existem dados de inquéritos epidemiológicos referentes à prevalência desse agravo na infância e adolescência que cubram todo o território nacional. Contudo, estudos pontuais em diversas regiões do país têm mostrado altas prevalências de hipertensão, oscilando entre $2,5 \%$ a $44,7 \%$ 7,8,9,10,11,12,13. Essa ampla variação pode decorrer, sobretudo, das diferentes metodologias empregadas, em especial no que diz respeito aos pontos de corte adotados e ao número de aferições da pressão realizada durante $o$ atendimento.

Dentre os fatores ambientais que concorrem para o aumento dos níveis da pressão arterial, destacam-se as mudanças associadas ao estilo de vida, em que se incluem hábitos alimentares inadequados associados à inatividade física, uso abusivo do álcool e tabagismo ${ }^{14}$. Estudos indicam que esses hábitos comportamentais estão correlacionados com a obesidade, um dos principais preditores da hipertensão arterial. Na infância, o excesso de peso tem sido identificado como uma epidemia global e, nas três últimas décadas, a prevalência da obesidade no Brasil em crianças e adolescentes passou de 11,4\%, em 1974-1975 15, para 33,4\%, em 2002-2003 16. Outros fatores de risco têm sido estudados na gênese da hipertensão arterial, a saber: a ausência do aleitamento materno 17 e o baixo peso ao nascer 18. Contudo, não é possível descartar o papel da escolaridade materna na adoção de comportamentos saudáveis, o que pode influenciar a magnitude da ocorrência desse agravo 19.

As doenças cardiovasculares são responsáveis por um terço das mortes no mundo, e a hipertensão arterial sistêmica é o fator de risco mais expressivo 20 . No Brasil, bem como na maior parte dos países desenvolvidos, as doenças cardiovasculares representam a principal causa de morbimortalidade e ocorrem atualmente em idades precoces, levando, consequentemente, à redução significativa da vida produtiva 21,22 . Assim, a detecção precoce dessas alterações pode contribuir para o desenvolvimento de programas de saúde de caráter preventivo, com enfoque na mudança do estilo de vida, voltada para a promoção da saúde, evitando-se que milhares de jovens desenvolvam prematuramente doença arterial coronariana e acidente vascular encefálico.

Portanto, o presente estudo teve como objetivo estimar a prevalência da pré-hipertensão e da hipertensão arterial, assim como avaliar os fatores associados à doença, em crianças e adolescentes matriculados em escolas públicas de Salvador, Bahia, Brasil.

\section{Métodos e técnicas}

Trata-se de um estudo transversal em que participaram estudantes com idade entre 7 e 14 anos, de ambos os sexos. Esses estudantes foram identificados em uma investigação mais ampla que teve por objetivo estudar fatores associados à anemia ferropriva em crianças e adolescentes matriculadas na rede pública de ensino da cidade de Salvador 23 .

O processo de amostragem no estudo original envolveu desenho complexo, valendo-se da estratificação das escolas em dois níveis (estadual e municipal), seguido pelo procedimento de amostragem por conglomerado em três estágios, conforme descrito a seguir: o primeiro estágio representado pelos distritos sanitários; o segundo, pelas escolas; o terceiro, pelos alunos. Em virtude de questões logísticas de campo, as informações dos estudantes selecionados foram extraídas de seis dos 12 distritos existentes em Salvador, cidade em que se observaram 117 escolas estaduais e 173 municipais. As escolas estaduais comportavam 58.059 alunos; as municipais, 56.555. Para atender ao número amostral previamente definido, verificou-se a necessidade de selecionar dez alunos de cada uma das 58 escolas municipais e 23 alunos de cada uma das 27 escolas estaduais, contabilizando-se 1.200 estudantes. Considerando que essa amostra não foi estimada, em face do objetivo investigado neste estudo, decidiu-se por calcular o erro amostral a posteriori. Nessas circunstâncias e com base na prevalência de níveis elevados de pressão arterial de 14\%, identificada neste estudo, o número amostral previamente adotado permitiu determinar os fatores associados ao desfecho estudado com erro de $2 \%$.

\section{Critério de exclusão}

Foram excluídas do estudo as adolescentes gestantes e nutrizes, além dos portadores de traumas físicos, que estavam imobilizados no momento das medições antropométricas.

\section{Coleta de dados}

\section{- Variável resposta: pressão arterial}

A pressão arterial das crianças e adolescentes foi aferida em dois momentos, com intervalo de dez minutos aproximadamente. A média entre as medidas representou a medida final utilizada para classificação da pressão arterial. Utilizou-se o esfigmomanômetro aneróide, calibrado no início da coleta dos dados por técnico especializado. A técnica para a aferição das medidas de pressão arterial seguiu as recomendações metodológicas das 
$V$ Diretrizes Brasileiras de Hipertensão Arterial 1. Para a realização das medidas, os aferidores foram submetidos a treinamento por supervisores experientes. Durante todo o trabalho de campo, os supervisores avaliavam periodicamente o desempenho dos aferidores e efetuava nova certificação do grupo a fim de minimizar possíveis erros associados à técnica auscultatória de verificação da pressão arterial.

Para a classificação da pressão arterial, foi inicialmente determinado o percentil da estatura/idade de cada estudante, com base na referência do Centers for Disease Control and Prevention dos Estados Unidos 24. De acordo com o percentil de estatura/idade, classificou-se a pressão arterial conforme o sexo, utilizando-se a recomendação do The Fourth Report on the Diagnosis, Evaluation, and Treatment of High Blood Pressure in Children and Adolescents 25 . Os critérios utilizados para classificação foram: pressão arterial normal (pressão arterial < percentil 90); pré-hipertensão (pressão arterial entre percentis 90 a 95); hipertensão estágio 1 (percentil 95 a 99mmHg); hipertensão estágio 2 (pressão arterial > percentil 99 mais $5 \mathrm{mmHg}$ ). Para análise dos dados, as categorias de hipertensão arterial estágio 1 e estágio 2 foram agregadas. Assim, a pressão arterial foi categorizada em normotensão (categoria de referência), pré-hipertensão e hipertensão (estágios 1 e 2).

\section{- Variáveis independentes: índice de massa corporal e circunferência da cintura}

As medidas antropométricas (peso e estatura) foram coletadas na escola, de maneira padronizada, seguindo os procedimentos preconizados pelo Anthropometric Standartization Reference Manual 26,27.

Com base nas medidas de peso e altura, foi calculado o índice de massa corporal (IMC) de todas as crianças e adolescentes. Para avaliar o estado antropométrico, foram utilizadas as tabelas de percentis da Organização Mundial da Saúde (OMS) como padrão de referência 28 . Para classificação, utilizou-se a proposta da OMS (2006): magreza ou baixo peso (< percentil 3), eutrofia ( $\geq$ percentil 3 e $<$ percentil 85 - categoria de referência), sobrepeso ( $\geq$ percentil 85 e $<$ percentil 97) e obesidade ( $\geq$ percentil 97). Para análise, foram agregadas as categorias sobrepeso e obesidade. Portanto, os indivíduos com excesso de peso apresentavam IMC situado no percentil igual ou acima de 85 .

A medida da circunferência da cintura foi realizada com fita métrica inelástica, graduada em centímetros e milímetros, seguindo procedimentos preconizados por Gillum et al. 29. A clas- sificação foi feita de acordo com os pontos de corte por idade e sexo apresentados no estudo de Taylor et al. ${ }^{30}$, que considera elevado valor acima do percentil 80 .

\section{Padrão de consumo alimentar}

O Questionário Quantitativo de Frequência Alimentar (QQFA) elaborado por Slater et al. 31 foi adaptado e adotado para avaliação da frequência de consumo alimentar dos estudantes pesquisados. Trata-se de um instrumento validado por Voci et al. 32. Constam do QQFA 132 itens alimentares, tendo sido adicionados alimentos regionais. A frequência de consumo desses itens foi dividida em quatro categorias, assim codificadadas: nunca consome $=0 ; 1$ a 3 vezes ao mês $=1$; 1 a 4 vezes na semana $=2 ; 5$ a 7 vezes na semana $=3$. Aqueles alimentos ou grupos de alimentos que apresentaram consumo acima de $50 \%$ pelos estudantes foram considerados para a realização da Análise Fatorial, sendo agrupados em 17 categorias, de acordo com semelhança de conteúdo nutricional (Tabela 1). Em seguida, foram somadas as frequências codificadas correspondentes aos alimentos efetivamente consumidos pelo indivíduo em cada grupo alimentar, o que constituiu o numerador da medida-resumo. $\mathrm{O}$ denominador correspondeu ao número máximo de alimentos que o indivíduo poderia consumir em cada grupo de alimentos multiplicado por 433 .

Antes de proceder à análise fatorial exploratória, o coeficiente Kaiser-Mayer-Olkin (KMO) foi estimado e o teste de esfericidade de Bartlett foi aplicado para indicar a adequação dos dados à análise 33 . Realizou-se a análise de componentes principais, seguida de uma rotação ortogonal (varimax), para examinar a estrutura (padrão) fatorial exploratória. O número de fatores a extrair foi definido conforme o gráfico da variância e pelo número de componentes (screen plot); os pontos no maior declive indicam o número apropriado de componentes a reter. A consistência interna das dimensões do QQFA foi avaliada, sendo considerado aceitável índice alpha de Cronbach $>0,65$. Foi realizada análise de Regressão Logística Politômica a fim de avaliar a associação dos padrões alimentares. Para tal, os escores gerados foram estratificados em duas categorias, tendo como ponto de corte a mediana.

\section{Nível de atividade física}

Para avaliação da atividade física, utilizou-se o Questionário de Atividades Físicas Realizadas Ontem (QUAFIRO). Trata-se de um instrumento desenvolvido por Russell R. Pate 34, da University of South Carolina (Estados Unidos), traduzido e 
Agrupamento dos alimentos usados na análise fatorial, conforme conteúdo nutritivo. Salvador, Bahia, Brasil, 2010.

\begin{tabular}{ll}
\hline Alimento ou grupo & Alimentos do questionário de frequência \\
\hline Leite & Leite em pó, iogurte \\
Pão & Pão branco, bolo, biscoito doce e salgado \\
Oleos & Margarina \\
Café & Café infusão \\
Refrigerante & Refrigerante \\
Feijão & Feijão \\
Embutidos & Embutidos \\
Pastelaria & Salgadinhos, salgado frito (quibe, pastel, coxinha etc.) \\
Peixe & Peixe \\
Frutas/Verduras & Quiabo, maçã, laranja, manga, maracujá, banana prata \\
Açúcares/Doces & Açúcar branco, sorvete, chocolate, geladinho, achocolatado \\
Carne de sol & Carne de sol \\
Carne bovina & Carne de boi fresca \\
Frango & Frango \\
Ovos & Ovo frito \\
Suco artificial & Suco artificial \\
Cereais & Arroz, batata, farinha de mandioca, macarrão \\
\end{tabular}

modificado por M. V. Nahas, do Núcleo de Pesquisa em Atividade Física e Saúde da Universidade Federal de Santa Catarina 35. O questionário informa a intensidade e o tempo gasto na atividade física. Com base nesses valores, obtém-se, então, o escore final do QUAFIRO multiplicandose o coeficiente da intensidade $(3,2$ ou 1 para intensa, moderada ou leve, respectivamente) pelo coeficiente da duração do exercício (1, 2 e 3 para < 15', 15'-30' e > 30', respectivamente). Neste estudo, o escore gerado foi estratificado em duas categorias, utilizando-se como ponto de corte a mediana: inativo (0-6 pontos) e ativo (igual ou acima de 7 pontos - categoria de referência).

\section{Características das condições ambientais e de moradia e socioeconômicas}

As características das condições ambientais e de moradia, socioeconômicas e maternas foram coletadas mediante questionários aplicados aos responsáveis pelos estudantes por entrevistadores treinados e qualificados. Os responsáveis foram convidados a comparecer na escola para as entrevistas. Foram obtidos dados acerca das características do domicílio (condições de posse do domicílio, tipo de construção, material predominante de piso, material predominante na cobertura e parte do domicílio, número de habitantes por dormitórios etc.) e de saneamento básico (abastecimento de água, coleta de lixo, esgotamento sanitário) para a construção de um índice adaptado do modelo proposto por Issler \& Giugliani 36. A cada situação, foi atribuída pontuação; a mais favorável recebeu o valor 0; a mais desfavorável, a pontuação 1. O somatório desses valores caracterizou o indicador das condições ambientais e de moradia. $\mathrm{O}$ índice foi classificado em dois estratos, tendo como ponto de corte a mediana: adequado (escore $\leq 4-$ categoria de referência) e inadequado (escore $>4$ ).

Foram coletados dados de renda familiar mensal. A renda foi traduzida em salário mínimo vigente $(\mathrm{R} \$ 380,00)$ no período do estudo, sendo categorizada em 3 estratos: < 1 salário mínimo; 1-3 salários mínimos e $\geq 3$ salários mínimos (categoria de referência).

\section{Características maternas}

Foram coletados dados de escolaridade materna. Para esta variável, foram considerados três níveis conforme as séries escolares cursadas: I - até o 5o ano; II - do 6o ao 9o anos e III - Ensino médio e superior (categoria de referência). Além disso, a idade materna foi classificada segundo a faixa etária: 10-19, 20-34 e 35 anos ou mais (categoria de referência).

\section{Análise dos dados}

Para o processamento e construção do banco de dados, foi utilizado o Epi Info versão 6.04 (Centers for Disease Control and Prevention, Atlanta, 
Estados Unidos), adotando-se a digitação dupla dos dados, após a revisão dos questionários e a correção dos erros decorrentes da codificação realizada inicialmente em campo.

A variável pressão arterial foi adotada como o desfecho. Para identificar os fatores que estão associados com a pré-hipertensão e hipertensão arterial, utilizou-se a análise de Regressão Logística Politômica. A magnitude da associação entre os fatores de risco e a ocorrência dos agravos (pré-hipertensão e hipertensão arterial), foi expressa em odds ratio (OR) e respectivos intervalos de confiança (IC95\%). O processo de modelagem foi baseado em estratégia ordenada da seguinte forma: primeiro, selecionadas as variáveis que apresentaram valor de $\mathrm{p} \leq 0,20$ na análise univariada politômica, conforme critério sugerido por Hosmer \& Lemeshow 37 ; posteriormente, realizada a análise multivariada empregando-se a técnica de Regressão Logística Politômica. Permaneceram no modelo ajustado apenas aquelas com valor de $\mathrm{p}<0,05$.

As análises estatísticas foram corrigidas pelo delineamento complexo da amostra, por meio da utilização do conjunto de comandos svy do Stata, versão 9.0 (Stata Corp., College Station, Estados Unidos).

\section{Questões éticas}

O projeto foi aprovado pelo Comitê de Ética do Instituto de Saúde Coletiva da Universidade Federal da Bahia, que apreciou e emitiu parecer favorável sobre a pertinência ética da investigação. Os pais ou responsáveis assinaram o termo de consentimento livre e esclarecido, autorizando a participação do filho na pesquisa.

\section{Resultados}

Do total de 1.200 estudantes inicialmente selecionados, registrou-se a perda de 69 deles (5,75\%), o que resultou em uma amostra de 1.131 participantes. Essas perdas se deveram à recusa em participar, à mudança da criança para outra cidade, ou transferência para outra escola. Desse total, seis estudantes foram excluídos em virtude da falta de registro de níveis pressóricos. Assim, a amostra efetivamente estudada constituiu-se de 1.125 crianças e adolescentes de ambos os sexos, com idade de 7 a 14 anos.

Foi detectada a prevalência de hipertensão de 4,8\% (IC95\%: 3,55-6,05), sendo mais pronunciada entre os estudantes do sexo feminino (sexo feminino: $7 \%$; sexo masculino $2,6 \%$ ) e entre aqueles com idade de 10 a 14 anos (10 a 14 anos: 5,6\%; 7 a 9 anos: 2,7\%). Detectou-se prevalência de pré-hipertensão de 9,3\% (IC95\%: 7,6-11,0), sendo mais pronunciada entre os do sexo feminino (sexo feminino: 10,1\%; sexo masculino $8,6 \%$ ) e entre aqueles com idade de 10 a 14 anos (10 a 14 anos: 9,9\%; 7 a 9 anos: 7,7\%). Evidenciou-se, ainda, que a ocorrência de sobrepeso/obesidade foi identificada em 12,6\% dos participantes; 8,7\% apresentaram circunferência da cintura acima do percentil 80; e 35,7\% eram fisicamente inativos (Tabela 2).

Tabela 2

Características demográficas, socioeconômicas e antropométricas, estilo de vida e história materna. Salvador, Bahia, Brasil, 2010 (N = 1.125).

\begin{tabular}{|c|c|c|}
\hline Variáveis & $\mathrm{n}$ & $\%$ \\
\hline \multicolumn{3}{|l|}{ Sexo } \\
\hline Masculino & 570 & 50,7 \\
\hline Feminino & 555 & 49,3 \\
\hline \multicolumn{3}{|l|}{ Idade (anos) } \\
\hline $7-9$ & 299 & 26,6 \\
\hline $10-14$ & 826 & 73,4 \\
\hline \multicolumn{3}{|l|}{ Estado antropométrico } \\
\hline Baixo peso & 100 & 8,9 \\
\hline Eutrofia & 883 & 78,5 \\
\hline Sobrepeso/Obesidade & 142 & 12,6 \\
\hline \multicolumn{3}{|l|}{ Circunferência da cintura } \\
\hline Adequado & 1.027 & 91,3 \\
\hline Inadequado & 98 & 8,7 \\
\hline \multicolumn{3}{|l|}{ Padrão de atividade física * } \\
\hline Inativo & 316 & 35,7 \\
\hline Ativo & 570 & 64,3 \\
\hline \multicolumn{3}{|c|}{ Indicador das condições ambientais e moradia ** } \\
\hline Adequado & 661 & 58,8 \\
\hline Inadequado & 463 & 41,2 \\
\hline \multicolumn{3}{|c|}{ Renda familiar total (salários mínimos) *** } \\
\hline III $-\geq 3$ & 143 & 12,8 \\
\hline$\|-1-3$ & 664 & 59,3 \\
\hline $\mid-<1$ & 312 & 27,9 \\
\hline \multicolumn{3}{|l|}{ Escolaridade materna \# } \\
\hline III - Ensino Médio e mais & 340 & 30,9 \\
\hline $11-6$ ㅇ-9으 anos & 406 & 36,8 \\
\hline 1 - até 5 o ano & 356 & 32,3 \\
\hline \multicolumn{3}{|l|}{ Idade materna (anos) \#\# } \\
\hline $20-34$ & 339 & 37,6 \\
\hline$\geq 34$ & 536 & 62,4 \\
\hline
\end{tabular}

* Dados faltantes: 239;

** Dado faltante: 1 ;

$\star \star \star$ Dados faltantes: 6 ;

\# Dados faltantes: 23;

\#\# Dados faltantes: 223. 
O índice de confiança da análise fatorial foi verificado por meio do coeficiente $\mathrm{KMO}(0,789)$, do teste de esfericidade de Bartlett $\left(\mathrm{c}^{2}{ }_{(1225)}=\right.$ 1586,496; $\mathrm{p}<0,001$ ) e do determinante da matriz de correlação $(0,351)$. Esses valores indicaram que as correlações entre os itens eram suficientes e adequadas para o processamento da análise fatorial. Desta análise, foram extraídos três padrões que representaram 44,53\% da variância total. Os padrões alimentares extraídos foram classificados como: padrão 1 - alimentos protetores: pão, grupo dos cereais, peixe e verduras/ frutas; padrão 2 - alimentos de risco: pastelaria (salgadinhos e salgados fritos), açúcares/doces (açúcar branco, sorvete, geladinho, achocolatado); padrão 3 - alimentos de risco: óleos e café (Tabela 3). Os índices referentes à consistência interna $(\mathrm{a}>0,60)$ garantiram aceitável nível de precisão de medida para os três padrões (Tabela 3 ), assegurando a consistência interna para as dimensões do questionário utilizado.

Tabela 3

Distribuição das cargas fatoriais dos padrões de consumo alimentar. Salvador, Bahia, Brasil, 2010.

\begin{tabular}{|c|c|c|c|c|}
\hline \multirow[t]{2}{*}{ Alimento ou grupo } & \multicolumn{3}{|c|}{ Padrão } & \multirow[t]{2}{*}{$h_{2}$} \\
\hline & 1으 & 2o & 3으 & \\
\hline Pão & 0,530 & - & - & 0,456 \\
\hline Pastelaria & - & 0,723 & - & 0,498 \\
\hline Óleos & - & - & 0,634 & 0,438 \\
\hline Açúcar/Doces & - & 0,678 & - & 0,484 \\
\hline Café & - & - & 0,713 & 0,449 \\
\hline Refrigerante & - & 0,669 & - & 0,512 \\
\hline Verduras/Frutas & 0,635 & - & - & 0,577 \\
\hline Peixe & 0,673 & - & - & 0,482 \\
\hline Cereais & 0,684 & - & - & 0,543 \\
\hline \% Variância explicada & 23,115 & 11,511 & 9,906 & - \\
\hline \% Variância acumulada & 23,115 & 34,626 & 44,532 & - \\
\hline
\end{tabular}

Nota: Significativos para o modelo: valores maiores que 0,50.

Observou-se, por meio de análise univariada (Tabela 4), associação positiva e estatisticamente significante entre pré-hipertensos e aqueles com sobrepeso/obesidade (OR: 3,20; IC95\%: 1,8115,656) e com a circunferência da cintura acima do percentil 80 (OR: 2,84; IC95\%: 1,038-5,543). Verificou-se, igualmente, associação positiva e estatisticamente significante entre hipertensão e sexo feminino (OR: 2,47; IC95\%: 1,287-4,773), a idade de 10-14 anos (OR: 2,53; IC95\%: 1,111-
5,791), os portadores de sobrepeso/obesidade (OR: 2,99; IC95\%: 1,510-5,921) e elevada circunferência da cintura (OR: 3,56; IC95\%: 1,694-7,507). Não houve associação para as demais variáveis estudadas.

Os fatores associados à pré-hipertensão e hipertensão estão apresentados na Tabela 5 (modelo final). Observou-se que a pré-hipertensão esteve associada somente ao sobrepeso/obesidade (OR: 3,13; IC95\%: 1,759-5,579). Já a hipertensão foi associada ao sexo feminino (OR: 2,49; IC95\%: 1,245-4,989), ao sobrepeso/obesidade (OR: 3,02; IC95\%: 1,455-6,284) e ao padrão alimentar de risco (OR: 1,93; IC\%: 1,045-3,569).

\section{Discussão}

A prevalência de 4,8\% de hipertensão encontrada entre as crianças e adolescentes matriculados na rede pública de Ensino Fundamental da cidade do Salvador é compatível com aquelas encontradas por Oliveira et al. 11 (3,6\%) em Feira de Santana, também no Estado da Bahia; por Rosa et al. 8 (4,6\%) em Niterói, Rio de Janeiro; por Monego \& Jardim 39 (5\%) em Goiânia, Goiás. Por outro lado, é maior que as identificadas por Borges et al. 39 (2,3\%) em Cuiabá, Mato Grosso; Rezende et al. 7 (2,5\%) em Barbacena, Minas Gerais; e menor do que aquelas encontradas por Xavier et al. 40 (13,5\%), em Uberaba, Minas Gerais; Gomes \& Alves 9 (17,3\%) em Recife, Pernambuco; e por Araújo et al. 10 (44,7\%) em Fortaleza, Ceará. Em relação à pré-hipertensão, observou-se, no presente estudo, prevalência de 9,6\%. Esse valor foi menor do que o revelado por Rosa et al. 8 $(8,6 \%)$, porém maior do que aquele encontrado por Borges et al. 39 (3,8\%). Deve-se ressaltar que ainda são escassos os estudos nacionais em que a pré-hipertensão é avaliada, apesar de ser conhecido o fato de que esta condição na infância está relacionada com o surgimento da hipertensão na vida adulta 5 .

A metodologia adotada para o diagnóstico de hipertensão arterial em cada estudo pode justificar a variabilidade observada. Fatores como a faixa etária estudada, número de visitas realizadas, número de aferições de pressão arterial em cada visita e o intervalo entre as aferições contribuem de forma importante para a variação de prevalências 14,41. É importante observar a falta de similaridade entre as metodologias utilizadas no diagnóstico de hipertensão em crianças e adolescentes, o que dificulta a comparação dos resultados dos estudos e pode implicar superestimação ou subestimação da prevalência.

Os resultados encontrados no estudo em questão mostraram que a hipertensão arterial 
Odds ratio bruta (OR) e respectivos intervalos de $95 \%$ de confiança (IC95\%) da associação de características sociodemográficas, antropométricas e maternas a pré-hipertensão e hipertensão arterial. Salvador, Bahia, Brasil, 2010 ( $N=1.125)$.

\begin{tabular}{|c|c|c|c|c|}
\hline \multirow[t]{2}{*}{ Variáveis } & \multicolumn{2}{|c|}{ Pré-hipertensão * } & \multicolumn{2}{|c|}{ Hipertensão * } \\
\hline & OR & IC $95 \%$ & OR & IC95\% \\
\hline \multicolumn{5}{|l|}{ Sexo } \\
\hline Masculino & 1,00 & & 1,00 & \\
\hline Feminino & $1,37 * *$ & $0,864-2,180$ & $2,47^{\star \star \star *}$ & $1,287-4,773$ \\
\hline \multicolumn{5}{|l|}{ Idade (anos) } \\
\hline $7-9$ & 1,00 & & 1,00 & \\
\hline $10-14$ & 1,26 & $0,732-2,170$ & 2,53 ** & $1,111-5,791$ \\
\hline \multicolumn{5}{|l|}{ Estado antropométrico } \\
\hline Eutrófico & 1,00 & & 1,00 & \\
\hline Baixo peso & 0,93 & $0,384-2,295$ & 0,67 & $0,198-2,299$ \\
\hline Sobrepeso/Obesidade & $3,20 * * *$ & $1,811-5,656$ & $2,99 * * \star$ & $1,510-5,921$ \\
\hline \multicolumn{5}{|l|}{ Circunferência da cintura } \\
\hline Adequado & 1,00 & & 1,00 & \\
\hline Inadequado & $2,84 * \star \star$ & $1,038-5,543$ & $3,56 * \star \star$ & $1,694-7,507$ \\
\hline \multicolumn{5}{|c|}{ Indicador das condições ambientais e moradia } \\
\hline Adequado & 1,00 & & 1,00 & \\
\hline Inadequado & 0,65 & $0,410-1,049$ & 0,54 & $0,295-1,017$ \\
\hline \multicolumn{5}{|c|}{ Renda familiar total (salários mínimos) } \\
\hline $\mid I I-\geq 3$ & 1,00 & & 1,00 & \\
\hline$\|-1-3$ & 0,58 & $0,296-1,126$ ** & 1,08 & $1,079-1,684$ \\
\hline $1-<1$ & 0,51 & $0,238-1,092$ ** & 1,21 & $0,407-3,629$ \\
\hline \multicolumn{5}{|l|}{ Escolaridade materna } \\
\hline III - Ensino Médio e mais & 1,00 & & 1,00 & \\
\hline 11 - 6ㅇ-9으 anos & 0,96 & $0,551-1,700$ & 1,02 & $0,494-2,135$ \\
\hline I - até 5o ano & 0,88 & $0,494-1,596$ & 0,98 & $0,472-2,033$ \\
\hline \multicolumn{5}{|l|}{ Idade materna (anos) } \\
\hline$\geq 35$ & 1,00 & & 1,00 & \\
\hline 20-34 & 0,65 & $0,378-1,132$ & 1,05 & $0,509-2,189$ \\
\hline \multicolumn{5}{|l|}{ Padrão de atividade física } \\
\hline Ativo & 1,00 & & 1,00 & \\
\hline Inativo & 0,76 ** & $0,570-1,017$ & 0,95 & $0,674-1,351$ \\
\hline \multicolumn{5}{|l|}{ Consumo alimentar } \\
\hline \multicolumn{5}{|c|}{ Padrão alimentar de proteção (escore) } \\
\hline Padrão 1 (> mediana) & 1,00 & & 1,00 & \\
\hline Padrão 1 (< mediana) & 0,89 & $0,563-1,423$ & 1,02 & $0,561-1,851$ \\
\hline \multicolumn{5}{|c|}{ Padrão alimentar de risco (escore) } \\
\hline Padrão 2 (< mediana) & 1,00 & & 1,00 & \\
\hline Padrão 2 (> mediana) & 1,20 & $0,758-1,914$ & 1,65 ** & $0,902-3,034$ \\
\hline \multicolumn{5}{|c|}{ Padrão alimentar de risco (escore) } \\
\hline Padrão 3 (< mediana) & 1,00 & & 1,00 & \\
\hline Padrão 3 (> mediana) & 0,95 & $0,600-1,511$ & 0,87 & $0,484-1,597$ \\
\hline
\end{tabular}

* Grupo de comparação: normotenso;

$\star * p<0,20$;

$* \star * p<0,05$. 
Odds ratio ajustada (OR) e respectivos intervalos de 95\% de confiança (IC95\%) para avaliar a associação entre pré-hipertensão, hipertensão e variáveis selecionadas. Salvador, Bahia, Brasil, 2010 ( $N=1.125)$.

\begin{tabular}{|c|c|c|c|c|}
\hline \multirow[t]{2}{*}{ Variáveis } & \multicolumn{2}{|c|}{ Pré-hipertensão * } & \multicolumn{2}{|c|}{ Hipertensão * } \\
\hline & OR & IC95\% & OR & IC95\% \\
\hline \multicolumn{5}{|l|}{ Sexo ** } \\
\hline Masculino & 1,00 & & 1,00 & \\
\hline Feminino & 1,35 & $0,845-2,171$ & 2,49 & $1,245-4,990$ \\
\hline \multicolumn{5}{|l|}{ Estado antropométrico ** } \\
\hline Eutrófico & 1,00 & & 1,00 & \\
\hline Baixo peso & 0,97 & $0,404-2,437$ & 0,74 & $0,213-2,595$ \\
\hline Sobrepeso/Obesidade & 3,13 & $1,759-5,579$ & 3,02 & $1,455-6,284$ \\
\hline \multicolumn{5}{|l|}{ Consumo alimentar ** } \\
\hline \multicolumn{5}{|c|}{ Padrão alimentar de proteção (escore) } \\
\hline Padrão 1 (> mediana) & 1,00 & & 1,00 & \\
\hline Padrão 1 (< mediana) & 0,88 & $0,552-1,419$ & 0,97 & $0,520-1,816$ \\
\hline \multicolumn{5}{|c|}{ Padrão alimentar de risco (escore) } \\
\hline Padrão 2 (< mediana) & 1,00 & & 1,00 & \\
\hline Padrão 2 (> mediana) & 1,29 & $0,805-2,084$ & 1,93 & $1,042-3,569$ \\
\hline \multicolumn{5}{|c|}{ Padrão alimentar de risco (escore) } \\
\hline Padrão 3 (< mediana) & 1,00 & & 1,00 & \\
\hline Padrão 3 (> mediana) & 1,06 & $0,661-1,698$ & 0,95 & $0,515-1,760$ \\
\hline
\end{tabular}

* Grupo de comparação: normotenso;

** Dados ajustados por renda.

esteve associada ao sexo feminino (OR: 2,49; IC95\%: 1,245-4,990). Nur et al. ${ }^{42}$ e Gomes \&Alves ${ }^{9}$, em seus estudos com crianças e adolescentes, observaram prevalências maiores de hipertensão entre aqueles indivíduos do sexo masculino. Borges et al. 39 e Ferreira \& Aydos 43 encontraram maiores prevalências entre os do sexo feminino. Portanto, os resultados dos estudos disponíveis não são consensuais para a distribuição do evento segundo o sexo.

A associação entre níveis pressóricos elevados e o estado antropométrico foi observada para o excesso de peso, que imprimiu chance 3,13 vezes maior de os participantes apresentarem pré-hipertensão arterial (OR: 3,13; IC95\%: $1,759-5,579)$ e chance 3,02 vezes maior de eles apresentarem hipertensão arterial (OR: 3,02; IC95\%: 1,455-6,284), quando comparados com os eutróficos. Essa associação é também relatada em inúmeros estudos epidemiológicos, nacionais e internacionais, tanto em adultos, quanto em crianças e adolescentes, confirmando uma unanimidade científica: a certeza de que o excesso de peso impacta negativamente os níveis pressóricos elevados 5,11,12,42,43,44,45,46.

Neste estudo, em análise univariada, verificou-se que circunferência da cintura está as- sociada a hipertensão arterial (OR: 3,56; IC95\%: 1,694-7,507) e pré-hipertensão (OR: 2,84; IC95\%: 1,038-5,543). Entretanto, para análise do modelo ajustado, em razão da forte associação entre as variáveis circunferência da cintura e IMC ( $\mathrm{p}<0,001$ ), optou-se pela entrada da variável IMC, já que grande parte dos estudos relatam a sua utilização 42,47. Há que se registrar, contudo, que, quando a circunferência da cintura foi utilizada em substituição ao IMC no modelo multivariado, observou-se associação positiva e estatisticamente significante entre a circunferência da cintura e pré-hipertensão (OR: 2,45; IC95\%: 1,278-4,707) e entre a mesma variável e hipertensão arterial (OR: 3,40; IC95\%: 1,484-7,823). Estudos mostram que a gordura localizada na região abdominal é um determinante de risco cardiovascular em crianças e adolescentes, sendo mais importante que o percentual de gordura corpórea 48 . Em estudo com adolescentes de 12 a 17 anos, Rosa et al. 49 observaram associação entre a hipertensão e a medida elevada de circunferência da cintura. Resultados semelhantes foram observados nos estudos de Guimarães et al. 50, Mariath \& Grillo 51 e Souza et al. 52, envolvendo crianças e/ou adolescentes. No entanto, os mecanismos fisiopatológicos que favorecem o de- 
senvolvimento de hipertensão na obesidade são complexos e multifatoriais. Dentre as alterações, destacam-se as hemodinâmicas sistêmicas e renais, resistência à insulina com hiperinsulinemia compensatória, ativação do sistema nervoso simpático e do sistema renina-angiotensina, além de efeitos da leptina plasmática 53,54,55.

O uso de análise fatorial com o método de extração dos componentes principais no QFA tem sido utilizado em diversos estudos para determinar os padrões alimentares 56,57,58,59. Dos três padrões identificados entre as crianças e adolescentes, um foi considerado saudável e dois potencialmente associados aos fatores de risco para doenças crônicas não transmissíveis. Destes dois, um abrange os alimentos pertencentes ao grupo das pastelarias (salgadinhos e salgados fritos - coxinhas, pastéis, quibes etc.) e ao dos açúcares/doces (açúcar branco, sorvete, geladinho, achocolatado); o outro é representado pelos óleos e café. Esse padrão de consumo reflete as modificações do estilo de vida vivenciado por crianças e adolescentes e pode ser explicado pela transição nutricional identificada na população brasileira nos últimos anos 16. Essas mudanças têm favorecido o aumento do consumo de alimentos industrializados, a alimentação fora de casa e a substituição das refeições tradicionais pelos lanches, levando ao consumo excessivo de sal, produtos gordurosos, açúcares simples, doces e bebidas açucaradas, além da diminuição do consumo de frutas, verduras e cereais integrais 60,61. Assim, o segundo padrão, constituído por alimentos de risco, imprimiu chance 1,93 vez maior de as crianças e adolescentes apresentarem hipertensão arterial (OR: 1,93: IC95\%: 1,042$3,569)$, quando comparada com a chance dos eutróficos.

Inexistem na literatura estudos que avaliem a relação entre hipertensão arterial e padrão alimentar em população infantil e em adolescente, utilizando a análise fatorial para identificar o perfil de consumo alimentar. Todavia, vários são aqueles que se utilizaram desta técnica de análise para investigar a associação de padrões dietéticos com fatores de risco para doenças cardiovasculares em adultos 33,59.

Com base na constatação do ritmo crescente das doenças crônicas não transmissíveis na infância e na adolescência, a OMS vem propondo, desde 2004, a adoção da Estratégia Global em Alimentação Saudável, Atividade Física e Saúde, que tem como objetivo reduzir os fatores de risco para as doenças crônicas não transmissíveis associadas a uma alimentação pouco saudável e aos baixos níveis de atividade física 20. Estudos recentes têm construído evidências de que mudanças positivas nos fatores modificáveis, em especial no padrão alimentar e no nível da atividade física dos indivíduos, respondem, em parte, pela redução da carga das doenças crônicas não transmissíveis para a população em geral 62 .

Diante disso, o sistema de saúde tem se organizado na tentativa de viabilizar intervenções voltadas para a promoção de um estilo de vida saudável, utilizando a escola como um dos espaços estratégicos para o diagnóstico, assim como para o incentivo à formação de hábitos alimentares saudáveis e à prática de atividades físicas regulares. Trata-se de uma iniciativa que foi adotada pelo Ministério da Saúde do Brasil, constituindo uma das diretrizes da Política Nacional de Alimentação e Nutrição do país 63 , recentemente contemplada como uma das diretrizes do Pacto pela Vida, em Defesa do SUS e de Gestão 64 . No entanto, embora as evidências indiquem que a adoção de estilo de vida saudável constitua a base fundamental para a prevenção e o tratamento dos fatores de risco para as doenças crônicas não transmissíveis, o desafio atual se constitui na execução de estratégias eficazes, duradouras e viáveis no campo da saúde pública que conduzam à adoção do estilo de vida saudável em crianças e adolescentes 62 .

É importante destacar as limitações deste estudo, impostas principalmente pelo seu desenho. Assim, salienta-se o fato de este ser um estudo transversal, o que limita a interpretação dos resultados, na medida em que, nesse tipo de estudo, não é possível estabelecer relações causais por não se contemplar a sequência temporal entre a exposição e o efeito. Por outro lado, os resultados encontrados são compatíveis com os registros da literatura consultada; dentre eles, alguns de intervenção, de que a melhoria dos hábitos alimentares e controle de peso constituem-se em importantes componentes na prevenção da hipertensão, entre outros fatores de risco associados às doenças cardiovasculares 65 .

Portanto, a despeito das limitações desta investigação, verificou-se que a prevalência de pré-hipertensão e hipertensão em crianças e adolescentes é maior entre aqueles com excesso de peso, do sexo feminino e com consumo alimentar inadequado. A detecção precoce dessas alterações (fatores modificáveis) pode favorecer a adoção de políticas e ações de controle dos fatores de risco associados a essa enfermidade. Sendo assim, é possível que o espaço escolar seja o ambiente favorecedor de ações de promoção de estilo de vida saudável, como alimentação adequada e atividade física, evitando que milhares de jovens desenvolvam prematuramente, em especial, doença arterial coronariana e vascular encefálica. 


\section{Resumo}

Trata-se de estudo para avaliar os fatores associados à hipertensão e pré-hipertensão. Participaram desta pesquisa 1.125 indivíduos com idade entre 7 e 14 anos da rede pública de ensino em Salvador, Bahia, Brasil. As variáveis de exposição foram: índice de massa corporal, circunferência da cintura, sexo, idade, atividade física, condições ambientais e de moradia, renda familiar, consumo alimentar, escolaridade e idade maternas. Os dados foram analisados utilizando-se a Regressão Logística Politômica e a variável desfecho categorizada em normotensos, pré-hipertensos e hipertensos. A prevalência de pressão arterial elevada foi de 14,1\%, sendo 4,8\% de hipertensão e 9,3\% préhipertensão. Houve associação entre pré-hipertensão e excesso de peso (OR: 3,13; IC95\%: 1,75-5,57). Hipertensão foi associada a excesso de peso (OR: 3,02; IC95\%: 1,45-6,28), sexo feminino (OR: 2,49; IC95\%: 1,24-4,98) e padrão alimentar de risco (OR: 1,93; IC95\%: 1,043,56). A prevalência de pré-hipertensão e hipertensão em crianças e adolescentes é maior entre aqueles com excesso de peso, do sexo feminino e com consumo alimentar inadequado.

Pré-hipertensão; Hipertensão; Fatores de Risco; Criança; Adolescente

\section{Referências}

1. Sociedade Brasileira de Cardiologia/Sociedade Brasileira de Hipertensão/Sociedade Brasileira de Nefrologia. V Diretrizes Brasileiras de Hipertensão Arterial. Arq Bras Cardiol 2007; 89:e24-79.

2. World Health Organization. Diet, nutrition and the prevention of chronic diseases. Report of a Joint WHO/FAO Expert Consultation. Geneva: World Health Organization; 2003.

3. World Health Organization. Issues of communication and risk. World Health Report 2002: from noncommunicable diseases \& mental health (NMH) communications. Geneva: World Health Organization; 2002.

4. Shear CL, Burke GL, Freedman DS, Berenson GS. Value of childhood blood pressure measurements and family history in predicting future blood pressure status: results from 8 years of follow-up in the Bogalusa Heart Study. Pediatrics 1986; 77:862-9.

5. Lauer RM, Clarke WR. Childhood risk factors for high adult blood pressure: the Muscatine Study. Pediatrics 1989; 84:633-4.

\section{Colaboradores}

S. L. Pinto participou da concepção do estudo, coleta e análise dos dados, interpretação dos resultados e redação do manuscrito. R. C. R. Silva participou da concepção do estudo, coleta e análise dos dados, interpretação dos resultados e redação do manuscrito. S. E. Priore participou da interpretação dos resultados e redação do manuscrito. A. M. O. Assis participou da concepção da interpretação dos resultados e redação do manuscrito. E. J. Pinto participou da análise dos dados, interpretação dos resultados e redação do manuscrito.

\section{Agradecimentos}

À Fundação de Amparo à Pesquisa do Estado da Bahia.
6. Li S, Chen W, Srinivasan SR, Bond MG, Tang R, Urbina EM, et al. Childhood cardiovascular risk factors and carotid vascular changes in adulthood: the Bogalusa Heart Study. JAMA 2003; 290:2271-6.

7. Rezende DF, Scarpelli RAB, Souza GF, Costa JO, Scarpelli AMB, Scarpelli PA, et al. Prevalência da hipertensão arterial sistêmica em escolares de 7 a 14 anos do Município de Barbacena, Minas Gerais, em 1999. Arq Bras Cardiol 2003; 81:375-80.

8. Rosa MLG, Fonseca VM, Oigman G, Mesquita ET. Pré-hipertensão arterial e pressão de pulso aumentada em adolescentes: prevalência e fatores associados. Arq Bras Cardiol 2006; 87:46-53.

9. Gomes BMR, Alves JGB. Prevalência de hipertensão arterial e fatores associados em estudantes de ensino médio de escolas públicas da Região Metropolitana do Recife, Pernambuco, Brasil, 2006. Cad Saúde Pública 2009; 25:375-81.

10. Araújo TL, Lopes MVO, Cavalcante TF, Guedes NG, Moreira RP, Chaves ES, et al. Análise de indicadores de risco para hipertensão arterial em crianças e adolescentes. Rev Esc Enferm USP 2008; 42:120-6. 
11. Oliveira AMA, Oliveira AC, Almeida MS, Almeida FS, Ferreira JBC, Silva CEP, et al. Fatores ambientais e antropométricos associados à hipertensão arterial infantil. Arq Bras Endocrinol Metab 2004; 48:849-54.

12. Silva MAM, Rivera IR, Ferraz MRMT, Pinheiro AJTP, Alves SWS, Moura AA, et al. Prevalência de fatores de risco cardiovasculares em crianças e adolescentes da rede de ensino da cidade de Maceió. Arq Bras Cardiol 2005; 84:387-92.

13. Borges MH, Viana FP, Rezende AD. Obesidade, doenças cardiovasculares e hipertensão. In: Dâmaso A, organizador. Obesidade. Rio de Janeiro: Editora Medsi; 2003. p. 64-101.

14. Salgado CM, Carvalhaes JTA. Hipertensão arterial na infância. J Pediatr (Rio J.) 2003; 79 Suppl 1: 115-24.

15. Instituto Brasileiro de Geografia e Estatística. Estudo Nacional de Despesas Familiares (ENDEF, 1974). Resultados preliminares. Brasília: Instituto Brasileiro de Geografia e Estatística; 1979.

16. Instituto Brasileiro de Geografia e Estatística. Pesquisa de Orçamentos Familiares/2002-2003. Antropometria e análise do estado nutricional de crianças e adolescentes no Brasil. Rio de Janeiro: Instituto Brasileiro de Geografia e Estatística; 2006.

17. Singhal A, Cole TJ, Lucas A. Early nutrition in preterm infants and later blood pressure: two cohorts after randomised trials. Lancet 2001; 357:413-9.

18. Barker DJP, Osmond C, Winter PD, Marguetts B. Weight in infancy and death from ischaemic heart disease. Lancet 1989; 2:577-80.

19. Monteiro CA, Conde WL. Time trends in overweight prevalence in children, adolescents and adults from less and more developed regions of Brazil. In: Ailhaud G, Guy-Grand B, editors. Progress in obesity research: 8th International Congress on Obesity. London: John Libby; 1999. p. 665-71.

20. World Health Organization. Global strategy on diet, physical activity and health Geneva: World Health Organization; 2003.

21. Grillo LP, Crispim SP, Siebert AN, Andrade ATW, Rossi A, Campos IC. Perfil lipídico e obesidade em escolares de baixa renda. Rev Bras Epidemiol 2005; 8:75-81.

22. Silva GAP, Balaban G, Motta MEFA. Prevalência de sobrepeso e obesidade em crianças e adolescentes de diferentes condições socioeconômicas. Rev Bras Saúde Matern Infant 2005; 5:23-9.

23. Borges CQ, Silva RC, Assis AMO, Pinto EJ, Fiaccone RL, Pinheiro SMC. Fatores associados à anemia em crianças e adolescentes de escolas públicas de Salvador, Bahia, Brasil. Cad Saúde Pública 2009; 25:877-88

24. Centers for Disease Control and Prevention/National Center for Health Statistics. CDC growth charts: United States, 2000. http://www.cdc.gov/ growthcharts (acessado em 20/Jan/2010).

25 . The fourth report on the diagnosis, evaluation, and treatment of high blood pressure in children and adolescents. National High Blood Pressure Education Program Working Group on High Blood Pressure in Children and Adolescents. Pediatrics 2004; 114:555-76.
26. Lohman TG, Roche AF, Martorell R. Anthropometric standardization reference manual. Champaign: Human Kinetics Books; 1988.

27. World Health Organization. Physical status: the use and interpretation of anthropometry. Geneva: World Health Organization; 1995.

28. Onis M, Onyango AW, Borghi E, Siyam A, Nishida C, Siekmann J. Development of a WHO growth reference for school-aged children and adolescents. Bull World Health Organ 2007; 85:660-7.

29. Gillum RF. Distribution of waist-to-hip ratio, other indices of body fat distribution and obesity and associations with HDL cholesterol in children and young adults aged 4-19 years: The Third National Health and Nutrition Examination Survey. Int J Obes Relat Metab Disord 1999; 23:556-63.

30. Taylor RW, Jones IE, Williams SM, Goulding A. Evaluation of waist circumference, waist-to-hip ratio, and the conicity index as screening tools for high trunk fat mass, as measured by dual-energy $\mathrm{X}$-ray absorptiometry, in children aged 3-19 y. Am J Clin Nutr 2000; 72:490-5.

31. Slater B, Fisberg RM, Philippi ST, Latorre MRO. Validation of a semi-quantitative adolescents food frequency questionnaire applied at a public school in São Paulo, Brazil. Eur J Clin Nutr 2003; 57: 629-35.

32. Voci SM, Enes CC, Slater B. Validação do Questionário de Frequência Alimentar para Adolescentes (QFAA) por grupos de alimentos em uma população de escolares. Rev Bras Epidemiol 2008; 11 : 561-72.

33. Neumann AICP, Martins IS, Marcopito LF, Araújo EAC. Padrões alimentares associados a fatores de risco para doenças cardiovasculares entre residentes de um município brasileiro. Rev Panam Salud Pública 2007; 22:329-39.

34. Pate RR. Physical activity assessment in children and adolescents. Crit Rev Food Sci Nutr 1993; 33:321-6.

35. Nahas MV. Atividade física, saúde e qualidade de vida: conceitos e sugestões para um estilo de vida ativo. 2a Ed. Londrina: Midiograf; 2001.

36. Issler RM, Giugliani ER. Identificação de grupos mais vulneráveis à desnutrição infantil pela medição do nível de pobreza. J Pediatr (Rio J.) 1997; 73:101-5.

37. Hosmer DW, Lemeshow S. Applied logistic regression. New York: John Wiley and Sons; 1989.

38. Monego ET, Jardim PCBV. Determinantes de risco para doenças cardiovasculares em escolares. Arq Bras Cardiol 2006; 87:37-45.

39. Borges LM, Peres MA, Horta BL. Prevalência de níveis pressóricos elevados em escolares de Cuiabá, Mato Grosso. Rev Saúde Pública 2007; 41:530-8.

40. Xavier RM, Xavier MM, Cartafina RA, Magalhães FO, Nunes AA, Santos VM. Prevalência de hipertensão arterial em escolares vinculados à universidade de Uberaba (UNIUBE). Brasília Méd 2007; 44:169-72.

41. Oliveira RG, Lamounier JA, Oliveira ADB, Castro MDR, Oliveira JS. Pressão arterial em escolares e adolescentes: o estudo de Belo Horizonte. J Pediatr (Rio J.) 1999; 75:256-66. 
42. Nur N, Cetinkaya S, Yilmaz A, Ayvaz A, Bulut MO, Sumer H. Prevalence of hypertension among high school students in a middle Anatolian province of Turkey. J Health Popul Nutr 2008; 26:88-94.

43. Ferreira JS, Aydos RD. Prevalência de hipertensão arterial em crianças e adolescentes obesos. Ciênc Saúde Coletiva 2010; 15:97-104.

44. Garcia FD, Terra AF, Queiroz AM, Correia CA, Ramos OS, Ferreira QT, et al. Avaliação de fatores de risco associados com elevação da pressão arterial em crianças. J Pediatr (Rio J.) 2004; 80:29-34.

45. He Q, Ding ZY, Fong DY-T, Karlberg J. Blood pressure is associated with body mass index in both normal and obese children. Hypertension 2000; 36:165-70.

46. Poletti OH, Barrios L. Obesidad e hipertensión arterial en escolares de la ciudad de Corrientes, Argentina. Arch Argent Pediatr 2007; 105:293-8.

47. Rademacher ER, Jacobs Jr. DR, Moran A, Steinberger J, Prineas RJ, Sinaiko A. Relation of blood pressure and body mass index during childhood to cardiovascular risk factor levels in young adults. J Hypertens 2009; 27:1766-74.

48. Daniels SR, Morrison JA, Sprecher DL, Khoury P, Kimball TR. Association of body fat distribution and cardiovascular risk factors in children and adolescents. Circulation 1999; 99:541-5.

49. Rosa ML, Mesquita ET, Rocha ER, Fonseca VM. Body mass index and waist circumference as markers of arterial hypertension in adolescents. Arq Bras Cardiol 2007; 88:573-8.

50. Guimarães ICB, Almeida AM, Santos AS, Barbosa DBV, Guimarães AC. Pressão arterial: efeito do índice de massa corporal e da circunferência abdominal em adolescentes. Arq Bras Cardiol 2008; 90:393-9.

51. Mariath AB, Grillo LP. Influência do estado nutricional, circunferência da cintura e história familiar de hipertensão sobre a pressão arterial de adolescentes. Rev Ciênc Méd 2008; 17:65-74.

52. Souza MGB, Rivera IR, Silva MAM, Carvalho ACC. Relação da obesidade com a pressão arterial elevada em crianças e adolescentes. Arq Bras Cardiol 2010; 94:714-9.

53. Ferrannini E, Buzzigoli G, Bonadonna R, Giorico MA, Oleggini M, Graziadei L, et al. Insulin resistance in essential hypertension. N Engl J Med 1987; 317:350-7.

54. Barroso SG, Abreu VG, Francischetti EA. A participação do tecido adiposo visceral na gênese da hipertensão arterial e doença cardiovascular aterôgenica. Um conceito emergente. Arq Bras Cardiol 2002; 78:618-30.
55. Rosa EC, Zanella MT, Ribeiro AB, Junior OK. Obesidade visceral, hipertensão arterial e risco cárdiorenal: uma revisão. Arq Bras Endocrinol Metab 2005; 49:196-204.

56. Nicklas TA, Webber LS, Thompson B, Berenson GS. A multivariate model for assessing eating patterns and their relationship to cardiovascular risk factors: the Bogalusa Heart Study. Am J Clin Nutr 1989; 49:1320-7.

57. Mishra G, Ball K, Arbuckle J, Crawford D. Dietary patterns of Australian adults and their association with socioeconomic status: results from the 1995 National Nutrition Survey. Eur J Clin Nutr 2002; 56:687-93.

58. Kerver JM, Yang EJ, Bianchi L, Song WO. Dietary patterns associated with risk factors for cardiovascular disease in healthy US adults. Am J Clin Nutr 2003; 78:1103-10.

59. Fung TT, Willett WC, Stampfer MJ, Manson JE, Hu FB. Dietary patterns and the risk of coronary heart disease in women. Arch Intern Med 2001; 161:1857-62.

60. Andrade RG, Pereira RA, Sichieri R. Consumo alimentar de adolescentes com e sem sobrepeso do Município do Rio de Janeiro. Cad Saúde Pública 2003; 19:1485-95.

61. Bertin RL, Klarkle ENL, Ulbrich AZ, Stabelini Neto A, Bozza R, Araujo IQ, et al. Estado nutricional e consumo alimentar de adolescentes da rede pública de ensino da cidade de São Mateus do Sul, Paraná, Brasil. Rev Bras Saúde Matern Infant 2008; 8:435-43.

62. Costa PRF, Assis AMO, Silva MCM, Santana MLP, Dias JC, Pinheiro SMC, et al. Mudança nos parâmetros antropométricos: a influência de um programa de intervenção nutricional e exercício físico em mulheres adultas. Cad Saúde Pública 2009; 25:1763-73.

63. Ministério da Saúde. Política nacional de alimentação e nutrição. Brasília: Ministério da Saúde; 2000.

64. Ministério da Saúde. Pacto pela vida, em defesa do SUS e de gestão. Brasília: Ministério da Saúde; 2006.

65. Kolasa KM. Dietary Approaches to Stop Hypertension (DASH) in clinical practice: a primary care experience. Clin Cardiol 1999; 22 (7 Suppl):III16-22.

Recebido em 26/Jun/2010

Versão final reapresentada em 16/Nov/2010 Aprovado em 28/Mar/2011 